\title{
Application Status and Prospect of Microwave Remote Sensing
}

\author{
Cheng Lele, Yan Xinsui, Zhou Mengqiu, Zhou Yongqin, Wang Hao, Wan Houzhao
}

Hubei University, Wuhan, Hubei, 430062

Keywords: Remote Sensing, Active Remote Sensing, Passive Remote Sensing

\begin{abstract}
With the development of space, electronics, information and other technology, human desire for the exploration of unknown things increasingly strong. The emergence of high-resolution microwave remote sensing technology is for all-weather observation of all-weather weather makes up for the shortcomings of other remote sensing technologies and gained widespread international attention. Based on the working principle of microwave remote sensing, this paper expounds the advantages of microwave remote sensing, then analyzes its research status and application in various fields, and draws the conclusion that microwave remote sensing has the characteristics of strong observing ability and wide application range. The development prospects of the technology were predicted.
\end{abstract}

\section{Introduction}

Microwave remote sensing mainly refers to the sensor through the various objects from the reflection, scattering or radiation of the microwave signal, its processing and processing after analysis to obtain the required information. Compared with visible light and infrared remote sensing technology, microwave remote sensing observation is very strong and the application field is very extensive. It can not only work around the clock, but also has a certain ability to penetrate, detect the internal information of objects. Synthetic Aperture Radar (SAR) is one of the most effective remote sensing in microwave remote sensing in recent 20 years. It can realize two-dimensional high-resolution active imaging. After half a century of development, it has become a mature remote sensing technology, But its application technology also has deepening the demand, more features and new system SAR is being developed.

In recent years, microwave remote sensing has been paid more and more attention by the state. Since 1970, China has been committed to the study of the principle and the test of ground equipment and airborne equipment. Through the successful launch of Shenzhou 4 and China Remote Sensing Satellite No. 1, Effective detection of data, which represents China has been achieved to support a variety of means of detection of spaceborne microwave remote sensing. State Council Information Office on December 27, 2016 issued the "2016 China Aerospace" white paper, clearly proposed to further enhance the level of satellite remote sensing system and basic product capacity to form continuous and stable business service capabilities, improve the space application service system, Promote industrial development. According to this goal, we have to solve the problem of microwave remote sensing service service steering, but also to strengthen the multi-mode optical observation, L-band differential SAR technology to further improve the microwave remote sensing system, and gradually form a satellite remote sensing technology global reception service capabilities , To promote space science, space technology, space applications in all-round development.

\section{Microwave Remote Sensing Works}

According to the different detection methods, microwave remote sensing can be divided into two categories, one is passive remote sensing, also known as passive remote sensing, the other is active remote sensing also known as active remote sensing.

Passive remote sensing refers to the direct reception of objects through the sensor heat radiation, and then through the corresponding mathematical physical model to analyze the physical and 
geometric properties of objects to identify objects. It is mainly used in microwave radiometer, it is a record for receiving from the measured object from the microwave emission of radiation information detector. As shown in Figure 1, the antenna of the microwave radiometer amplifies the signal after receiving the signal, and then amplifies the amplified signal through the mixer to the middle frequency band, and then after the IF filter to further amplify the signal, and then detected by the detector, Finally using the integrator smooth output signal.

Active remote sensing is through the electromagnetic wave devices such as antennas, etc., to the target after the launch of electromagnetic waves in the form of echo after the remote sensing detection. According to the use of echo signals will be active remote sensing system is divided into two categories, the first category is the distance measurement system, the second category is the scattering system. The distance measurement system is to study the time delay of the transmitted and received signals to measure the distance such as radar altimeter, etc. The scattering system is mainly to study the strength and phase of the received signal, such as microwave scatterometer, real aperture radar (SLR), synthetic aperture radar SAR) and so on.

The microwave radar altimeter is designed to achieve distance detection by measuring the time delay between the transmitted signal and the received signal. The operation principle shown in Figure 2, the pulse signal generator to the electromagnetic wave signal to the antenna, and then by the antenna to the space and the Earth's surface launch, while the antenna also as a receiving antenna to receive echo signals from the Earth's surface, and the signal Transmitted to the processing equipment for results analysis. This is also the common operating characteristics of all active remote sensing devices. SAR is a high-resolution two-dimensional imaging radar, which, on the basis of SLR, uses the remote sensing platform to replace the increase in the size of the antenna and increases the equivalent length without changing the actual length of the antenna. "Synthetic aperture".

\section{Microwave Remote Sensing Advantage}

Microwave due to its unique electromagnetic wave band makes microwave remote sensing has other remote sensing technology unparalleled advantages.

1) can work all day, all day. Cloud rain and snow mist on the microwave transmittance has little effect, so any bad weather on the impact of microwave transmission are not large, which often have a large number of clouds covering the Earth's surface is undoubtedly a significant impact.

2) have a certain ability to penetrate the ground. Microwave compared to visible light and infrared on the ground has a certain ability to penetrate, to provide information within the object, so microwave remote sensing can be used for geological and mineral exploration and underground military building detection.

3) Microwave remote sensing can be used for interferometric measurements. Active microwave remote sensing can use the electromagnetic wave phase information to calculate the elevation of a point, its accuracy can reach several meters, this is the interference measurement. Using this technique, microwave remote sensing can monitor geological changes (such as earthquakes and crustal movements).

4) have special spectral characteristics for certain features. Many of the features in the microwave band between the radiation and scattering capacity is very different, so the use of their microwave spectral characteristics can be relatively easy to distinguish the target.

5) microwave remote sensing can be multi-polarization measurement. Microwave in different polarized state of the target to detect the information is not the same, so microwave remote sensing can also use microwave different polarization state detection target to get more information.

In addition, compared with the infrared range distance is short, ultrasonic ranging there is a blind spot, microwave remote sensing in the distance also has obvious advantages (measurement distance, high precision, fast response). According to the above analysis, microwave remote sensing on the features and space have a strong ability to observe. 


\section{Research Status and Its Application}

International microwave remote sensing technology has been developed for more than 50 years, it has gone from land-based to airborne to the development of the process of spaceborne, has become the main means of observation and space observation, nearly half of the Earth orbit satellite and deep space exploration Devices are equipped with microwave remote sensing equipment. In the international microwave remote sensing technology, the most rapid development and application of the most remote sensor is SAR. SARs used in aeronautical applications can achieve a resolution of less than 1 meter, even in low visibility weather conditions can still be similar to the photographic high resolution radar images. Launched SAR satellites include: Radarsat-2 in Canada, SAR-Lupe and TerraSAR in Germany, and TecSAR in Israel are all technologically advanced SAR satellites [1]. Multi-polarization, multi-band and optimized load are the main research directions of SAR. At the same time, microwave radiometer has received more and more attention in the world, and it is the main load of many meteorological satellites. For example, GeoSTAR, which is funded by the NASA IIIP project since 2002, uses two-dimensional integrated aperture radiometer for meteorological applications.

With the continuous innovation and development of microwave remote sensing technology and its unique superiority, microwave remote sensing has a wide range of applications, covering a wide range of fields such as agriculture and geology, oceanography, atmosphere and space, in order to study global dynamics and monitoring and prevention disaster.

China is a large agricultural country, crop cultivation type, area, scope and its yield valuation on the country to take economic policy has a huge impact. The use of microwave remote sensing can be the type of crop identification and planting range extraction, the growth of crops and health status, soil moisture and waterlogging monitoring, in order to regulate crop cultivation, food management, and timely measures to resist natural disaster.

Microwave penetration makes microwave remote sensing technology can be used to detect geological structure information, analysis of microwave images to obtain a variety of mineral rock, oil, natural gas and metal sediment information. The geological disasters such as debris flow can be identified and evaluated by SAR data 19 of different phases. The InSAR (Interference Synthetic Aperture Radar) data can be used to calculate the ground elevation after earthquakes, thus assessing the extent and extent of the earthquake. The Wenchuan earthquake in 2008 and the Qinghai earthquake in 2010 brought life-threatening and huge losses to the local population, and the weather was complex, but the SAR data were not affected by the cloud and rain, so the earthquake disaster can be monitored and evaluated.

Ocean area accounts for $70.8 \%$ of the Earth's area, it not only has a wealth of energy, but also affect the global climate, so we must profoundly understand and understand the ocean. In the August 10, 2016 launch of the "high score three" satellite can be day and night, regardless of climate monitoring of the ocean, available from the sea surface surface wind, sea level, sea, sea height and ocean surface temperature and other Accurate data, improve the accuracy of marine environmental parameters and sea conditions forecast, so that we can develop and protect marine resources, and conducive to law enforcement rights. SAR has an extremely important role in monitoring oil spills. When SAR detects oil spills, the nearest government can take immediate action to reduce losses.

Seawater will form sea ice at low temperatures, and the melting of sea ice affects the global climate and the survival of Antarctic organisms. Flow ice will endanger sailing ships and offshore structures, so many countries have started monitoring sea ice earlier. In 2003, Kang Jiancheng et al in the Antarctic sea ice scientific investigation, the first time the sea ice physical temperature machine snow cover, in order to determine the distribution and coverage of sea ice to provide accurate data. Scholars use SAR data to study sea ice thickness [2]. The study shows that the cross-polarization data of C-band and X-band SAR have high sensitivity to the thickness of first-born sea ice and perennial sea ice, so the relevant remote sensing physical model The thickness of the sea ice is retrieved from this data.

Atmospheric remote sensing plays a very important role in the development of atmospheric 
sciences, and its accurate prediction and forecast for severe weather conditions allows us to take protective measures in a timely manner to reduce losses. Compared with visible light, microwave can penetrate the atmosphere, the use of microwave remote sensing can detect different levels of atmospheric oxygen, water vapor and other trace gas distribution data for the study of atmospheric temperature and humidity distribution. Monitoring of hail can use ground-based radars to monitor the development phase of hail by the different radar echo characteristics. Tropical storms are often formed in tropical tropics in summer, and typhoons are formed when the storm accumulates to a certain extent. The effects of real-time monitoring on the effects of typhoon clouds and winds can be observed by microwave remote sensing.

In August 10, 2016, "high score three" successfully launched, which is China's first resolution of 1 meter C-band multi-polarized SAR imaging satellites, is the world's most work of SAR satellites, remove The previous band imaging mode and scanning imaging mode, but also increased the beam, wave, global observation, high and low incidence angle 10 kinds of imaging mode, can be used to monitor the ocean and ground dynamics, to achieve a multi-purpose function. Remote sensing technology in the earth observation and space exploration is essential, 2016 launch of the Temple II also loaded a number of ground observation remote sensor. As early as 2007 launched the first lunar artificial satellite "Chang'e One" for the first time equipped with microwave radiation remote sensing system, can be used to detect the lunar surface soil thickness and its internal structure.

China's microwave remote sensing technology has achieved many results, penetrate all walks of life, in the satellite exploration, marine environmental monitoring, climate monitoring, agricultural cultivation and fisheries development play an important role. At the same time microwave remote sensing has also been applied to all aspects of our lives, such as weather forecasting, agricultural planting, land use planning, environmental protection and natural disaster prediction, for human life has brought great convenience.

\section{Conclusion}

At present, the further development of microwave remote sensing and its application research is still in progress, microwave remote sensing is also facing some urgent problems to be solved. Such as SAR imaging will appear geometric distortion phenomenon, and to obtain a higher resolution using continuous imaging technology will lead to image spots and affect the observation. But with the image processing and interpretation of the technology, these problems will be gradually resolved. On the other hand, the development of microwave passive remote sensing applications is still constrained by the low spatial resolution. The main methods to improve the spatial resolution are to study the integrated aperture microwave radiometer and fully polarized microwave radiometer. China in 2001 and 2004 successfully developed two integrated aperture microwave radiometer, indicating that China has basically mastered the interference of synthetic aperture microwave imaging of the key technologies. As well as full polarization microwave radiometer, microwave passive remote sensing technology is also the focus of development.

Microwave remote sensing is one of the most challenging high-tech fields in the world today. Our microwave remote sensing technology has also passed through for more than 40 years. In the past 40 years, with the development of space, electronics and information technology, microwave remote sensing technology has been developed rapidly, spaceborne microwave remote sensing has been successfully applied to ground observation and space exploration, China's microwave remote sensing technology Catch up with the international level.

Microwave remote sensing has now formed a relatively complete discipline system and technology system, its application is more and more widely, but also led to the development of some cutting-edge technology, resulting in a significant social and economic benefits. It is conceivable that, in the near future, microwave remote sensing can better serve China's security, economic and social development. 


\section{Acknowledgements}

Fund Project: Natural Science Foundation of Hubei Province Youth Fund Project No. 2016CFB102

\section{References}

[1] Sun Jia. Analysis of Development Trend of Foreign Synthetic Aperture Radar Satellite [J]. Journal of Equipment Command \& Technology College, 2007 (01): 67-70.

[2] J W Kim, D J Kim, B J Hwang. Estimation of sea ice thickness in the Arctic Sea using polarimetric parameters of C-and X-band space-borne SAR date [C]. IGARSS, 2010 